\title{
A STUDY TO COMPARE EFFECTS OF SHOULDER POSITION ON INFRACLAVICULAR CENTRAL VENOUS CATHETERIZATION IN CRITICALLY ILL PATIENTS.
}

1. MBBS, FCPS

Assistant Professor Anesthesia \& Critical Care

SZMC Rahim Yar Khan, Pakistan 2. MBBS, FCPS

Registrar Coronary Care Unit SZMC Rahim Yar Khan, Pakistan.

Correspondence Address:

Dr. Sairah Sadaf

Department of Anesthesia \& Critical Care

SZMC Rahim Yar Khan, Pakistan. sairahbabar@live.com

Article received on: 13/01/2019

Accepted for publication: 03/02/2020

\begin{abstract}
Sairah Sadaf', Babar Bashir ${ }^{2}$
ABSTRACT... Objectives: Aim of this study was to compare the effects of two different shoulder positions on infraclavicular subclavian venous catheterization in critically ill patients. Study Design: Prospective Comparative study. Setting: Sheikh Zayed Medical College Rahim Yar Khan Pakistan. Period: $1^{\text {st }}$ July 2017 to $30^{\text {th }}$ June 2018. Material \& Methods: Enrolling 100 patients thru convenient sampling, divided into two groups, Group A \& Group B, 50 in each group. In group A patients were put in supine position, with head turned to contra lateral side and caudal pull was applied on ipsilateral shoulder. While in group B patients were lying supine, head turned to contra lateral side and shoulders were retracted by placing a small pillow vertically under the chest between the scapulae. In both groups, subclavian vein was approached through infra clavicular route. Number of attempts of venous punctures (1st attempt / 2nd attempt), total time spent on procedure (from 1st skin puncture to CVC insertion), complications (arterial puncture, pneumothorax, hydrothorax, malpositioning), any hemodynamic irregularity (ECG changes), radiographic findings to confirm successful CVC insertion was recorded. Results: Number of successful subclavian venous catheterizations was same in both groups (94\% vs. $94 \%)$ with no significant difference $(p=1.000) .1^{\text {st }}$ attempt success was more in group $A$ as compared to group $B$, though statistically this was not significant $(p=0.275)$. Total time spent on CVC insertion (from $1^{\text {st }}$ skin puncture to catheter insertion) was less than 05 mints in $84 \%$ patient in group A and $82 \%$ in group B. While more than 05 mints were spent on $16 \%$ vs. $18 \%$ patients in group A vs. B. This was also not statistically significant $(p=0.790)$. In group A 2/50 (4\%) while in group B 1/50 (2\%) cases were recorded as malpositioning on post-CVC radiograph. Other complications were not encountered in either group. Conclusion: Lowered shoulder and retracted shoulder positions are equally effective for SVC insertion in terms of success, $1^{\text {st }}$ attempt success, total time spent and number of complications.
\end{abstract}

Key words: Catheterization, Classic, Infra Clavicular, Lowered, Retracted, Subclavian.

Article Citation: Sadaf S, Bashir B. A study to compare effects of shoulder position on infraclavicular central venous catheterization in critically ill patients. Professional Med J 2020; 27(10):2216-2222.

DOI: 10.29309/TPMJ/2020.27.10.4483

\section{INTRODUCTION}

CVC (central venous catheter) is more often in use as high quality care not only in peri-operative period but also in critical care. ${ }^{1}$ In 1929 , Werner Fossman was the first physician who introduced CVC devices. ${ }^{2,3}$ Sven-Ivar Seldinger, in 1950, used a novel technique for percutaneous CVC placement, known as "Seldinger Technique", which is now widely in medical practice. ${ }^{4}$ CVC has numerous indications such as trauma resuscitation, hemodynamic monitoring, total parentral nutrition, drug administration, hemodialysis, plasma pheresis, cardiopulmonary resuscitation, rapid fluid resuscitation and pace maker insertion..$^{5-8}$ As with any other invasive procedure, CVC insertion is also associated with some mechanical complications such as pneumothorax, haemothorax, failure to puncture vein, Arterial puncture ${ }^{9}$ and malpositioning. ${ }^{10,11}$

CVC insertion is a bedside procedure that is practiced in a number of ways, adopting different central veins and/or patient positions, according to physician's choice e.g. internal jugular, subclavian and femoral veins. Subclavian venous catheterization can be performed via supra or infraclavicular routes, amongst which infraclavicular route is more popular. ${ }^{9,12}$ On the 
other hand, subclavian vein is the most popular one as compared to internal jugular vein for catheterization, because of its association with lesser infectious complications ${ }^{13-15}$ \& better tolerance by critical care patients. ${ }^{16,17} \mathrm{~A}$ study conducted by Sandhu et al reflects that as compare with subclavian vein, internal jugular venous catheterization was more frequently associated with inadvertent arterial punctures and poor tolerance by patients. ${ }^{18}$

Taking in account of central venous choices and patient positions, a lot of differences are found among many previous studies. Raju S. et al has compared neutral shoulder position and lowered shoulder position in his study, which has reflected superiority of neutral shoulder position for subclavian VC insertion thru infraclavicular route. ${ }^{4}$ Similarly another study conducted by Tarbiat $\mathrm{M}$. et al in Iran shows results which confirms success rate of neutral arm position vs. ipsilateral abducted arm position for infraclavicular subclavian VC insertion $96.2 \%$ vs $84.4 \%$ respectively. ${ }^{12}$ Another study conducted by Fortune JB et al in US, has compared Trendelenburg position, retracted shoulder position and head turned position for subclavian VC insertion. Their results show superiority of Trendelenburg position over other two positions. ${ }^{6}$

Due to discrepancies in results of previous studies, a single best position has not been established for infraclavicular subclavian vein catheterization until now. No study was conclusive in terms of which position or maneuver is best for subclavian venous puncture. As CV catheterization is an invasive procedure, subjected to many complications if performed without comprehension and competency. So we wanted to conduct a study to compare different shoulder positions while performing this invasive procedure to reach on a conclusion in our setup with limited resources. It will definitely help our interns and doctors in critical care to learn a safe, efficient and quick method for CVC insertion thus reducing morbidity and mortality in critically ill patients.

\section{Objective}

To compare the effects of two different shoulder positions on infraclavicular subclavian venous catheterization in critically ill patients.

Null hypothesis: Lowered shoulder and retracted shoulder positions are equally effective in subclavian VC insertion thru infra clavicular route.

Alternate Hypothesis: Lowered shoulder position is more effective than retracted shoulder position for subclavian VC insertion thru infra clavicular route.

\section{MATERIAL \& METHODS}

This prospective comparative study, after approval from ethical committee, was conducted at Sheikh Zayed Medical College Rahim Yar Khan Pakistan, in a period from $1^{\text {st }}$ July 2017 to $30^{\text {th }}$ June 2018 , in Critical care and Coronary care units. By keeping confidence level $95 \%$ and power of $80 \%$, on the basis of a previous study ${ }^{12}$, sample size of 95 patients was calculated, which was rounded of to 100 . Patients were taken in the study through convenient sampling, aged between 15 years to 85 years. Written informed consent was taken either from patients or the relatives of ventilator dependent patients. Patients were divided into two groups $A$ \& $B, 50$ patients in each group as follow:

\section{Group A}

Patients were put in supine position, with caudal traction on ipsilateral arm to lower down shoulder, with head rotation to contra lateral side.

\section{Group B}

Patients were put in supine position, head turned to contra lateral side and shoulders were retracted by placing a small pillow vertically under the chest between scapulae.

Right subclavian venous catheterization through infraclavicular approach.

Modified Seldinger's technique was applied for infraclavicular subclavian venous catheter insertion by experienced coronary and critical care physicians, having experience of more than 
10 years in said field. Exclusion criteria were significant coagulopathy, local infection and chest deformity.

As patients were in critical care and coronary care units, all the necessary monitoring was already attached to the patients along with a working peripheral venous line. Patients were already sedated and paralyzed on ventilators as per protocol, while patients in coronary care unit were sedated with inj. Midazolam, dosage according to body weight and local anesthetic was infiltrated on the puncture side with $2 \%$ xylocain. All aseptic measures were taken. A $15 \mathrm{~cm}$ double-lumen CVC (of B-Braun $20 \mathrm{~cm}$, made in Germany) was used.

Patients of group A were lying supine with slight caudal traction on ipsilateral arm by an assistant to lower down the shoulder, while in patients of group B a small pillow was placed under the chest between scapulae. In both groups patient's head slightly turned to contra lateral side. After sterile preparation, skin was punctured $1 \mathrm{~cm}$ below the middle and medial junction of clavicle with the puncture needle attached to a syringe, provided in the kit. Needle's direction was towards suprasternal notch making $10^{\circ}$ angle with the skin, while its bevel was directed downwards. Needle was advanced in the said direction until it touched the clavicle. Maintaining slight negative pressure, needle was further advanced under the inferior boarder of clavicle very slowly until the right subclavian vein was punctured. After confirming non-pulsatile venous blood, syringe was removed and the guide wire was passed with J-tip directing downwards. Dilator was passed over the guide wire to slightly dilate the venous track. Double lumen CVC was then slid over the guide wire and guide wire was removed afterwards. All ports of central line were checked for free flow of blood and air bubbles were removed. CVC was then fixed to the skin with suture and ASD was applied. In case of $1^{\text {st }}$ attempt failure, puncture needle was withdrawn until its tip was in subcutaneous tissue and needle was forwarded in slightly cephalic or caudal direction. Placement was considered successful when the CVC tip was in distal superior vena cava
(SV) or at the junction of SV and right atrium on radiograph. CVC tips found in ipsilateral Internal Jugular vein or contra lateral brachiocephalic vein were labeled as malpositioned catheters. Other complications such as pneumothorax were also excluded through post-insertion chest radiographs.

Data was collected on a Performa which includes age, gender, body weight, identification of group ( $\mathrm{A}$ or $\mathrm{B})$, number of attempts of venous puncture $\left(1^{\text {st }}\right.$ attempt $/ 2^{\text {nd }}$ attempt), total time spent on procedure (from $1^{\text {st }}$ skin puncture to CVC insertion), complications (arterial puncture, pneumothorax, hydrothorax, malpositioning), any hemodynamic irregularity (ECG changes), radiographic findings to confirm successful CVC insertion.

Data was entered in SPSS version 16. Quantitative data was represented by mean + SD and median and qualitative data was presented as frequency and percentages. Association between qualitative variables was assessed by Chi-square test. While quantitative data between the two groups was analyzed by using t-test. Results were also represented in graphical forms where necessary. The $\mathrm{P}<0.05$ was considered as significant.

\section{RESULTS}

We studied 100 patients, 50 in each group (A \& B); Group A with caudal pull on ipisilateral shoulder, while Group B with retracted shoulders. According to data there were $34(68 \%)$ males and $16(32 \%)$ females in group A while $24(48 \%)$ males and 26(52\%) females in group B (Table-I) \& (Figure-1). This difference was significant $(p=0.043)$. Age between the two groups was also significantly different $(p=0.013)$ as $52.88+16.7$ years vs. $44.20+17.5$ years in group-A and group-B respectively (Table-II \& Figure-2). While there was no significant difference in body weight between the two groups (Table-II) \& (Figure-2).

\begin{tabular}{|c|c|c|c|c|}
\hline Gender & Group A & Group B & Total & \multirow{4}{*}{$\begin{array}{c}P= \\
0.043\end{array}$} \\
\hline Male & $68 \%(34)$ & $48 \%(24)$ & $58 \%(58)$ & \\
\hline Female & $32 \%(16)$ & $52 \%(26)$ & $42 \%(42)$ & \\
\hline Total & $100 \%(50)$ & $100 \%(50)$ & $100 \%(100)$ & \\
\hline
\end{tabular}




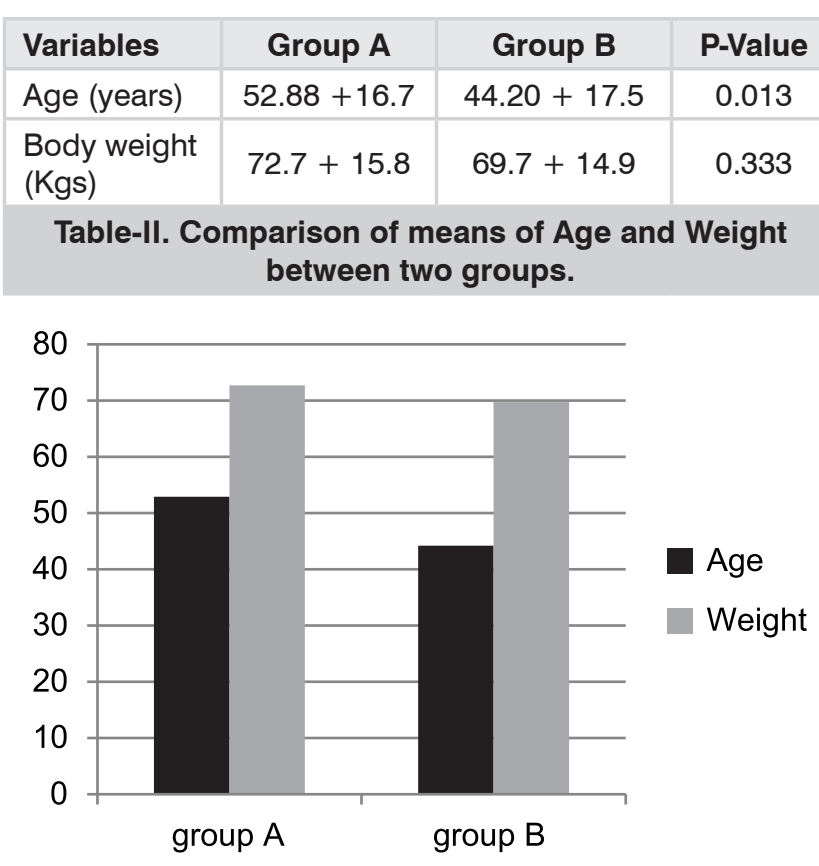

Figure-1. Gender distribution in two groups.

Number of successful subclavian venous catheterizations was same in both groups $(94 \%$ vs. $94 \%)$ with no significant difference $(p=1.000)$ (Table-III). Number of failed catheterizations was $6 \%$ in both groups. $1^{\text {st }}$ attempt successful catheterization achieved was $88 \%$ (44/50) vs. $80 \%(40 / 50)$ while number of $2^{\text {nd }}$ attempt was $12 \%$ (6/50) vs. $20 \%$ (10/50) in group A \& B respectively (Table-III). $1^{\text {st }}$ attempt success was more in group A as compared to group $B$, though statistically this was not significant $(p=0.275)$ (Figure-3). Total time spent on CVC insertion (from $1^{\text {st }}$ skin puncture to catheter insertion) was less than 05 mints in $84 \%$ patient in group $A$ and $82 \%$ in group $\mathrm{B}$ (Table-III). While more than 05 mints were spent on $16 \%$ vs. $18 \%$ patients in group A vs. B. This was also not statistically significant $(p=0.790)$.

\begin{tabular}{|c|c|c|c|c|}
\hline \multicolumn{2}{|c|}{ Variables } & Group A & Group B & $\begin{array}{c}\text { P. } \\
\text { Value }\end{array}$ \\
\hline \multicolumn{2}{|c|}{ Success } & $94 \%(47 / 50)$ & $94 \%(47 / 50)$ & \multirow{2}{*}{1.000} \\
\hline \multicolumn{2}{|c|}{ Failure } & $6 \%(3 / 50)$ & $6 \%(3 / 50)$ & \\
\hline \multicolumn{2}{|c|}{$1^{\text {st }}$ attempt } & $88 \%(44 / 50)$ & $80 \%(40 / 50)$ & \multirow{2}{*}{0.275} \\
\hline \multicolumn{2}{|c|}{$2^{\text {nd }}$ attempt } & $12 \%(6 / 50)$ & $20 \%(10 / 50)$ & \\
\hline \multirow{2}{*}{$\begin{array}{l}\text { Time } \\
\text { spent }\end{array}$} & $<5$ mints & $84 \%(42 / 50)$ & $82 \%(41 / 50)$ & \multirow{2}{*}{0.790} \\
\hline & $>5 \mathrm{mints}$ & $16 \%(8 / 50)$ & $18 \%(9 / 50)$ & \\
\hline
\end{tabular}

Table-III. Comparing Success, number of $1^{\text {st }} \& 2^{\text {nd }}$ attempt and time spent between two groups.

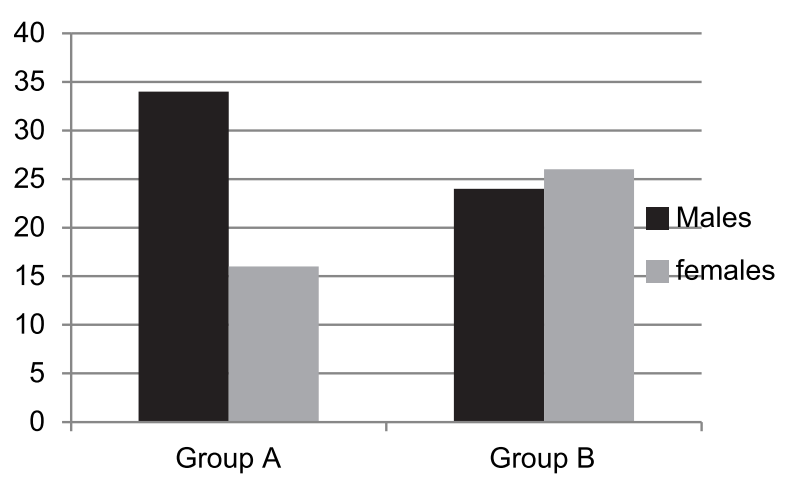

Figure-2. Age \& weight comparison in two groups.

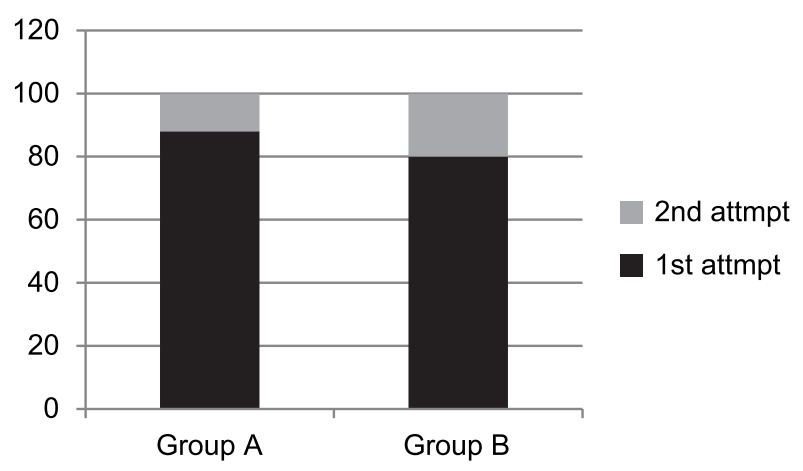

Figure-3. comparing number of attempts in two groups.

As far as complications were concerned such as arterial puncture, pneumothorax and hydrothorax not a single case was found $(0 \%$ complications in either group) (Table-IV). While 1 case was found to have an episode of benign cardiac arrhythmias on cardiac monitor, during CVC insertion in group B (Table-V). On slight with drawl of CVC, arrhythmia was corrected immediately without any serious consequences. In group A 2/50 (4\%) while in group B 1/50 (2\%) cases were recorded as malpositioning on post-CVC radiograph (into ipsilateral RIJV in group $A$ and into contra lateral brachiocephalic vein in group-B).

\begin{tabular}{|l|c|c|}
\hline \multicolumn{1}{|c|}{ Variables } & Group A & Group B \\
\hline Pneumothorax & $0 \%$ & $0 \%$ \\
\hline Hydrothorax & $0 \%$ & $0 \%$ \\
\hline Arterial Puncture & $0 \%$ & $0 \%$ \\
\hline \multicolumn{2}{|c|}{ Table-IV. Comparing complications between the } \\
groups.
\end{tabular}




\begin{tabular}{|l|c|c|c|}
\hline Variables & Group A & Group B & P-Value \\
\hline Malpositioning & $4 \%(2 / 50)$ & $2 \%(1 / 50)$ & 0.558 \\
\hline Cardiac Arrhythmia & $0 \%$ & $2 \%(1 / 50)$ & 0.315 \\
\hline \multicolumn{2}{|c|}{ Table-V. Comparing complications between the } \\
groups.
\end{tabular}

\section{DISCUSSION}

While catheterizing subclavian vein thru infra clavicular route, one should be certain about the anatomical changes produced by different shoulder positions. A single best shoulder position for SVC insertion has been probed by researchers, but results so far are not conclusive. The "Classic Position", for subclavian venous catheterization thru infraclavicular route, is the position of shoulders with a pillow/roll in longitudinal direction, underneath chest between the scapulae (retracted shoulders). ${ }^{19,20}$ Some other shoulder positions, like "neutral shoulder position" and "lowered shoulder position" (with caudal pull on ipsilateral arm), have also been under investigation, yielding a variety of results.

Literature reveals that retracted shoulders (classic position), may relocate the right SV more medially in relation to clavicle, which may lead to aberrant placement of CV catheters. Whereas lowered shoulder position may produce a more constant relationship between SV and clavicle thus leading to proper and efficient CVC placement. However this may also decrease the SV diameter. Yet there are some studies which reflect that "Classic position" decreases the diameter of subclavian vein thus making it more difficult to cannulate the vein thru infra clavicular approach..$^{21,22}$

There is found radiological evidences such as magnetic resonance imaging (MRI) and Ultrasonography (USG), demonstrating that, as a matter of fact, the diameter of subclavian vein decreases by retraction of shoulders. ${ }^{21,22}$ Fortune JB et al has studied 05 different positions under USG for subclavian VC and they have concluded that shoulder retraction and head turning to opposite side may attenuate target size and provide an undesirable position for subclavian puncture. ${ }^{6}$ Their results favor the neutral shoulder and head position. Another USG guided study, done by Rodriguez CJ et al in 2007, has produced similar results like Fortune JB, that when patient is lying supine or in Trendelenburg position, the retracted shoulders may significantly decrease the cross sectional area of subclavian vein. ${ }^{22}$

After these drastic findings in contrast to the traditional practice of "classic position", we meticulously evaluated the optimal shoulder position for SVC insertion in our study. Our results are contrasting to the available data, revealing that there is statistically no difference between retracted and lowered shoulder positions, success was same in both groups $(p=1.000)$.

Kang $M$ et al studied effects of neutral and lowered shoulder positions on SCV insertion and their results have demonstrated superiority of neutral shoulder position over lowered shoulder position. ${ }^{23}$ In their study $1 / 173$ and 2/173 SV catheterizations were failed in neutral vs. lowered positions respectively, which are comparable to our results ( 3 failed cases in both groups). This difference was statistically insignificant. Catheter tip malpositioning in their study was more in lowered shoulder group $8 \%$ vs. $1.15 \%$, which is again consistent with our results (malpositioning of CVC more in lowered shoulder group $4 \%$ vs. $2 \%$ ). This relocation of $\mathrm{CV}$ catheter can be explained on the anatomical fact that the lowered shoulder position can attenuates the angle between SV and brachiocephalic vein which may lead to ipsilateral IJV catheter placement.

A study conducted by Raju $S$ et al, evaluating effects of neutral vs. lowered shoulder position on SCV insertion ${ }^{4}$, has demonstrated more successful SCV catheterizations (96.7\% vs. 93.3\%) in neutral group vs. lowered shoulder group respectively that is statistically not significant. Number of failed catheterizations was $1 / 30$ and $2 / 30$ in neutral vs. lowered shoulder groups respectively which was consistent with our results. In their study $1^{\text {st }}$ attempt success to catheterize was only $46.6 \%$ in lowered shoulder group in contrast to $60 \%$ in neutral group. These findings are inconsistent with our study, where $1^{\text {st }}$ attempt success was more in lowered shoulder group vs. retracted shoulder group (88\% vs. $80 \%$ respectively). In 
their study Arterial puncture was more (10\%) in lowered shoulder group as compared to neutral group (6.6\%). While in our study there was no arterial puncture in either group.

Another study conducted by Kim $\mathrm{HJ}$ et $\mathrm{al}^{24}$, comparing neutral position with retracted shoulder position on SCV insertion, reveals statistically insignificant results in terms of success $(95.6 \%$ vs. $96.1 \%$ respectively). According to their study complications rate was also statistically same in both groups, although number of arterial punctures was high in retracted shoulder group. Pneumothorax and SVC malpositioning was same in both groups. These findings are not in concordance with our study results, where number of SVC malpositioning was less in retracted shoulder group as compare to lowered shoulder position ( $2 \%$ vs. $4 \%$ respectively). There was no arterial puncture or pneumothorax in both of our study groups.

Recently more studies are being done with ultrasound guidance but at our center with restricted resources it is not feasible. So we have to use surface landmarks to continue with this invasive critical procedure at our center.

\section{CONCLUSION}

There is no single best position for SV catheterization as all positions have their own risks and benefits. According to our study results it is found that lowered shoulder and retracted shoulder positions are equally effective for SVC insertion in terms of success, $1^{\text {st }}$ attempt success, time duration and number of complications. So both positions can be practiced for SVC insertion as both are efficient equally.

\section{LIMITATIONS}

There are some limitations to our study like sample size should be more. We used convenient sampling technique which is a probable limitation of this study. Further studies are required with a large sample size under ultrasound guidance. Copyright(C) 03 Feb, 2020.

\section{REFERENCES}

1. Agarwal S, Payal YS, Sharma JP. A retrospective clinical audit of 696 central venous catheterizations at a tertiary care teaching hospital in India. J Emerg Trauma Shock. 2012; 5(4):304-308.

2. Beheshti MV. A concise history of central venous acess. Tech Vasc Interv Radiol. 2011; 14(4):184-185.

3. Buckley CJ, Buckley SD. Evolution of endovascular therapy: Where are we now? In: Khanna NN, Henry $M$,editors. Handbook of interventions for structural heart and peripheral vacular disease. New Delhi: Jaypee; 2016, p. 95-8.

4. Raju S, Ubale P, Nasir AS. A study to evaluate the influence of shoulder position on central venous catheter tip location during infraclavicular subclavian approach. Anesthesia, Pain \& Intensive Care. 2016; 20(4):393-397.

5. Scalea TM, Sinert R, Duncon AO, et al. Percutaneous central venous access for resuscitation in trauma. Acad Emerg Med. 1994; 1:525-531.

6. Fortune $\mathrm{Jb}$, Feustel P. Effect of patient on size and location of the subclavian vein for percutaneous puncture. Arch Surg. 2003; 138:996-1000.

7. Czarnik T, Gawda R, Perkowski T, et al. Supraclavicular approach is easy and safe method of subclavian vein catheterization even in mechanically ventilated patients: Analysis of $\mathbf{3 7 0}$ attempts. Anesthesiology. 2009; 111:334-339.

8. Tarbiat M, Farhanchi F, Davoudi M, et al. Supraclavicular vs infraclavicular subclavian catheterization in coronary artery bypass graft surgery. Res Cardiovasc Med. 2018; 7(1):5-9.

9. Mansfield PF, Hohn DC, Fornage BD, etal. Complications and failures of subclavian-vein catheterization. $\mathrm{N}$ Engl J Med. 1994; 331:1735-1738.

10. Webb JG, Simmonds SD, Chan-Yan C. Central venous malpositions presenting as chest pain. Chest. 1986; 89:309-312.

11. Yilmazlar A, Bilgin $H$, Koorfali $G$, et al. Complications of 1303 central venous cannulations. J R Soc Med. 1997; 90:319-321.

12. Tarbiat $M$, Davoudi $M$, Salimbahrami SA. Influence of arm position during infraclavicular subclavian vein catheterization in coronary artery bypass graft surgery. J Cardiovasc Thorac Res. 2018; 10(4):192196. 
13. Linos DA, Mucha P, Van-Heerden JA. Subclavian vein: A golden route. Mayo Clin Proc. 1980; 55(5):315-321.

14. Thompson EC, Calver LE. Safe subclavian vein cannulation. Am Surg. 2005; 71:180-183.

15. Ruesch S, Walder B, Tramer MR. Complications of central venous catheters: Internal Jugular versus Subclavian access - A systemic review. Crit Care Med. 2002; 30:454-460.

16. Kocum A, Sener M, Caliskan E,et al. An alternative central venous route for cardiac surgery: supraclavicular subclavian vein catheterization. J Cardiothorac Vasc Anesthesia. 2011; 25(6):1018-1023.

17. Tarbiat M, Manafi B, Davoudi M, et al. Comparison of the complications between left side and right side subclavian vein catheterization. J Cardiovasc Thorac Anesthesia. 2014; 6(3):147-151.

18. Sandhu NS. Transpectoral ultrasound guided catheterization of axillary vein: An alternative to standard catheterization of subclavian vein. Anesth Analg. 2004; 99:183-187.
19. McGee DC, Gould MK. Preventing complications of central venous catheterization. N Engl J Med. 2003; 348:1123-1133.

20. Taylor RW, Palagiri AV. Central venous catheterization. Crit Care Med. 2007; 35:1390-1396.

21. Jesseph JM, Conces DJ Jr, Augustyn GT. Patient positioning for subclavian vein catheterization. Arch Surg. 1987; 122:1207-1209.

22. Rodriguez CJ, Bolanowski A, Patel K, et al. Classical positioning decreases the cross-sectional area of the subclavian vein. Am J Surg. 2006; 192:135-137.

23. Kang M, Ryu HG, Son IS, et al. Influence of shoulder position on central venous catheter tip location during infraclavicular subclavian approach. British J of Anesth. 2011; 106(3):344-347.

24. Kim HJ, Jung SH, Min J, et al. Comparison of neutral and retracted shoulder positions for infraclavicular subclavian venous catheterization: A randomized, non-inferiority trial. British $J$ of Anesth. 2013; 111(2):191-196.

\begin{tabular}{|c|c|c|c|}
\hline \multicolumn{3}{|c}{ AUTHORSHIP AND CONTRIBUTION DECLARATION } \\
\hline Sr. \# & Author(s) Full Name & \multicolumn{1}{|c|}{ Contribution to the paper } & Author(s) Signature \\
\hline 1 & Sairah Sadaf & $\begin{array}{l}\text { Conception, deisgn of work, analysis } \\
\text { of data, interpretation of resutls, } \\
\text { Drafting of manuscript, final approval, } \\
\text { Accountable for all espects of work. } \\
\text { Conception, Revising, Participation in } \\
\text { particile conduct, Final approval. }\end{array}$ \\
\hline 2 & Babar Bashir & \\
\hline
\end{tabular}

